American J. of Engineering and Applied Sciences 1 (2): 161-167, 2008

ISSN 1941-7020

(C) 2008 Science Publications

\title{
Nonlinear Whitham-Broer-Kaup Wave Equation in an Analytical Solution
}

\author{
S.A. Zahedi, M.Vaezi and N. Tolou \\ Department of Mechanical Engineering, \\ Mazandaran University, Babol, P.O. Box 484, Iran
}

\begin{abstract}
This study presented a new approach for the analysis of a nonlinear Whitham-Broer-Kaup equation dealing with propagation of shallow water waves with different dispersion relations. The analysis was based on a kind of analytical method, called Variational Iteration Method (VIM). To illustrate the capability of the approach, some numerical examples were given and the propagation and the error of solutions were shown in comparison to those of exact solution. In clear conclusion, the approach was efficient and capable to obtain the analytical approximate solution of this set of wave equations while these solutions could straightforwardly show some facts of the described process deeply such as the propagation. This method can be easily extended to other nonlinear wave equations and so can be found widely applicable in this field of science.
\end{abstract}

Key words: Variational iteration method (VIM), propagation of wave equations, whitham-broer-kaup equation

\section{INTRODUCTION}

Most of scientific problems and phenomena occur nonlinearly. Except a limited number of these problems, most of them do not have precise analytical solutions so that these have to be solved using other methods. Many different new methods have recently presented; for example, Variational Iteration Method $(\mathrm{VIM})^{[1-3]}$, Homotopy Perturbation Method (HPM ${ }^{)^{[4-8]}}$ Homotopy Analysis Method ${ }^{[9]}$ and Exp function ${ }^{[10]}$.

In this article, VIM is used to solve nonlinear equations of Whitham-Broer-Kaup with the initial conditions $u(x, 0)=F(x), v(x, 0)=G(x)$ for the initial time.

$$
\begin{gathered}
\mathrm{u}_{\mathrm{t}}+\mathrm{uu}_{\mathrm{x}}+\mathrm{v}_{\mathrm{x}}+\beta \mathrm{u}_{\mathrm{xx}}=0 \\
\mathrm{v}_{\mathrm{t}}+(\mathrm{uv})_{\mathrm{x}}+\alpha \mathrm{u}_{\mathrm{xxx}}-\beta \mathrm{v}_{\mathrm{xx}}=0
\end{gathered}
$$

If $\alpha=0, \beta \neq 0$, system (1) becomes classical long wave equation that describe shallow water wave with dispersive $^{[11]}$. If $\alpha=1, \beta=0$, system (1) becomes variant Boussinesq equation. In this research we will focus on finding analytical approximate and exact traveling wave solution of the system (1) using the variational iteration method.

\section{MATERIALS AND METHODS}

Fundamental of variational iteration method: To clarify the basic ideas of VIM, we consider the following differential equation ${ }^{[1-3]}$ :

$$
\mathrm{Lu}+\mathrm{Nu}=\mathrm{g}(\mathrm{t})
$$

where, $\mathrm{L}$ is a linear operator, $\mathrm{N}$ a nonlinear operator and $\mathrm{g}(\mathrm{t})$ an inhomogeneous term.

According to VIM, we can write down a correction functional as follows ${ }^{[1-3]}$ :

$$
u_{n+1}(t)=u_{n}(t)+\int_{0}^{t} \lambda\left(L u_{n}(\tau)+N \tilde{u}_{n}(\tau)-g(\tau)\right) d \tau
$$

where, $\lambda$ is a general Lagrangian multiplier which can be identified optimally via the variational theory. The subscript $\mathrm{n}$ indicates the 9th approximation and $\tilde{\mathrm{u}}_{\mathrm{n}}$ is considered as a restricted variation $\delta \tilde{u}_{\mathrm{n}}=0$.

Implementation of Variational Iteration Method: We first consider the application of VIM to WBK Eq. 1 subject to the following initial conditions ${ }^{[11]}$ :

$$
\begin{gathered}
\mathrm{u}(\mathrm{x}, 0)=\omega-2 \mathrm{Bk} \operatorname{coth}(\mathrm{k} \xi) \\
\mathrm{v}(\mathrm{x}, 0)=-2 \mathrm{~B}(\mathrm{~B}+\beta) \mathrm{k}^{2} \operatorname{csch}^{2}(\mathrm{k} \xi)
\end{gathered}
$$

Corresponding Author: S.A. Zahedi, Department of Mechanical Engineering, Mazandaran University, Babol, P.O. Box 484, Iran 
where, $\mathrm{B}=\sqrt{\alpha+\beta^{2}}$ and $\xi=\mathrm{x}+\mathrm{x}_{0}$ and $\mathrm{x}_{0}, \mathrm{k}, \omega$ are arbitrary constants.

Its correction variational function in $\mathrm{x}$ and $\mathrm{t}$ can be expressed respectively as follows:

$$
\begin{aligned}
& u_{n+1}(x, t)=u_{n}(x, t)+\int_{0}^{t} \lambda_{1}(\tau)\left\{\begin{array}{c}
\dot{u}_{n}+u_{n} u_{n}^{\prime} \\
+v_{n}^{\prime}+\beta u_{n}^{\prime \prime}
\end{array}\right\} d \tau \\
& v_{n+1}(x, t)=v_{n}(x, t)+\int_{0}^{t} \lambda_{2}(\tau)\left\{\begin{array}{c}
\dot{v}_{n}+\left(u_{n} v_{n}\right)^{\prime} \\
+\alpha u_{n}^{\prime \prime \prime}-\beta v_{n}^{\prime \prime}
\end{array}\right\} d \tau
\end{aligned}
$$

where, prime indicates a differential with respect to $\mathrm{x}$ and dot denotes a differential with respect to $t, \lambda_{1}$ and $\lambda_{2}$ are general Lagrangian multipliers.

After some calculations, we obtain the following stationary conditions:

$$
\begin{array}{r}
\lambda_{1}^{\prime}(\tau)=0 \\
1+\left.\lambda_{1}(\tau)\right|_{\tau=\mathrm{t}}=0 \\
\lambda_{2}^{\prime}(\tau)=0 \\
1+\left.\lambda_{2}(\tau)\right|_{\tau=\mathrm{t}}=0
\end{array}
$$

Equation $7 \mathrm{a}$ and $8 \mathrm{a}$ are called Lagrange-Euler equations and $\mathrm{Eq} .7 \mathrm{~b}$ and $8 \mathrm{~b}$ are natural boundary conditions.

The Lagrange multipliers can therefore, be identified as $\lambda_{1}=\lambda_{2}=-1$ and the variational iteration formula is obtained in the form of:

$$
\begin{aligned}
& u_{n+1}(x, t)=u_{n}(x, t)-\int_{0}^{t}\left\{\dot{u}_{n}+u_{n} u_{n}^{\prime}+v_{n}^{\prime}+\beta u_{n}^{\prime \prime}\right\} d \tau \\
& v_{n+1}(x, t)=v_{n}(x, t)-\int_{0}^{t}\left\{\dot{v}_{n}+\left(u_{n} v_{n}\right)^{\prime}+\alpha u_{n}^{\prime \prime \prime}-\beta v_{n}^{\prime \prime}\right\} d \tau
\end{aligned}
$$

We start with the initial approximation of $u(x, 0)$ and $\mathrm{v}(\mathrm{x}, 0)$ given by Eq. 4 . Using the above iteration formulas 9 and 10, the other components can be obtained directly as follows:

$$
\begin{aligned}
& \mathrm{u}_{0}(\mathrm{x}, \mathrm{t})=\omega-2 \mathrm{Bk} \operatorname{coth}(\mathrm{k} \xi) \\
& \mathrm{v}_{0}(\mathrm{x}, \mathrm{t})=-2 \mathrm{~B}(\mathrm{~B}+\beta) \mathrm{k}^{2} \operatorname{csch}^{2}(\mathrm{k} \xi)
\end{aligned}
$$

$$
\begin{aligned}
\mathrm{u}_{1}(\mathrm{x}, \mathrm{t})= & -\left[-\omega \sinh (\mathrm{k} \xi) \cosh ^{2}(\mathrm{k} \xi)+\omega \sinh (\mathrm{k} \xi)\right. \\
& +2 \mathrm{Bk} \cosh ^{3}(\mathrm{k} \xi)-2 \mathrm{Bk} \cosh (\mathrm{k} \xi)+ \\
& \left.2 \mathrm{Bk}^{2} \mathrm{t} \omega \sinh (\mathrm{k} \xi)\right] \\
/ & \left\{\left[\cosh ^{2}(\mathrm{k} \xi)-1\right] \sinh (\mathrm{k} \xi)\right\} \\
\mathrm{v}(\mathrm{x}, \mathrm{t})= & 2 \mathrm{Bk}[-\cosh (\mathrm{k} \xi) \mathrm{B}+\mathrm{B}-\cosh (\mathrm{k} \xi) \beta+\beta \\
& +4 \mathrm{Bk} \mathrm{t} \cosh (\mathrm{k} \xi)+2 \mathrm{Bkt} \\
& -2 \mathrm{Bk} \cosh (\mathrm{k} \xi) \mathrm{t} \omega \sinh (\mathrm{k} \xi) \\
& -2 \mathrm{k} \cosh (\mathrm{k} \xi) \mathrm{t} \omega \beta \sinh (\mathrm{k} \xi) \\
& -4 \alpha \mathrm{tk} \cosh (\mathrm{k} \xi)-2 \alpha \mathrm{tk} \\
& -4 \beta \mathrm{tk} \cosh (\mathrm{k} \xi)-2 \beta \mathrm{tk}] \\
/[ & \cosh (\mathrm{k} \xi)-1]
\end{aligned}
$$

In the same manner, the rest of the components of the iteration formula can be obtained.

$$
\begin{aligned}
& u(x, t)=u(x, t)-\left\{\dot{u}+u u^{\prime}+v^{\prime}+\beta u^{\prime \prime}\right\} d \tau \\
& v(x, t)=v(x, t)-\left\{\dot{v}+(u v)^{\prime}+\alpha u^{\prime \prime \prime}-\beta v^{\prime \prime}\right\} d \tau
\end{aligned}
$$

Exact solution of WBK Eq. 1 is as follows:

$$
\begin{gathered}
u(x, t)=\omega-2 B k \cosh [k(\xi-\omega t)] \\
v(x, t)=-2 B(B+\beta) k \operatorname{csch}[k(\xi-\omega t)]
\end{gathered}
$$

These solutions are constructed by ${ }^{[12]}$.

Special case one: As a special case, if $\alpha=1$ and $\beta=0$, WBK Eq. 1 can be reduced to the Modified Boussinesq (MB) Eq. 19:

$$
\begin{aligned}
& u+u u+v=0 \\
& v+(u v)+u=0
\end{aligned}
$$

Solving these equations by VIM as follows:

$$
\begin{aligned}
& \mathrm{u}(\mathrm{x}, \mathrm{t})=\mathrm{u}(\mathrm{x}, \mathrm{t})+\lambda(\tau)\left\{\dot{\mathrm{u}}+\mathrm{u} \mathrm{u}^{\prime}+\mathrm{v}^{\prime}\right\} \mathrm{d} \tau \\
& \mathrm{v}(\mathrm{x}, \mathrm{t})=\mathrm{v}(\mathrm{x}, \mathrm{t})+\lambda(\tau)\left\{\dot{\mathrm{v}}+(\mathrm{uv})^{\prime}+\mathrm{u}^{\prime \prime}\right\} \mathrm{d} \tau
\end{aligned}
$$

We consider the initial conditions of the MB Equations $^{[11]}$ : 


$$
\begin{aligned}
& \mathrm{u}(\mathrm{x}, 0)=\omega-2 \mathrm{k} \operatorname{coth}(\mathrm{k} \xi) \\
& \mathrm{v}(\mathrm{x}, 0)=-2 \mathrm{k} \operatorname{csch}(\mathrm{k} \xi)
\end{aligned}
$$

The Lagrange multipliers can therefore, be identified as $\lambda_{1}=\lambda_{2}=-1$ and the variational iteration formula is obtained in the following form:

$$
\begin{aligned}
& u(x, t)=u(x, t)-\left\{\dot{u}+u u^{\prime}+v^{\prime}\right\} d \tau \\
& v(x, t)=v(x, t)-\left\{\dot{v}+(u v)^{\prime}+u^{\prime \prime}\right\} d \tau
\end{aligned}
$$

We are to start with an initial approximation $\mathrm{u}(\mathrm{x}, 0)$ and $\mathrm{v}(\mathrm{x}, 0)$ given by Eq. 22 , by the above iteration formula 23 and 24.We can obtain directly the other components as follows:

$$
\begin{gathered}
\mathrm{u}(\mathrm{x}, \mathrm{t})=\omega-2 \mathrm{k} \operatorname{coth}(\mathrm{k} \xi) \\
\mathrm{v}(\mathrm{x}, \mathrm{t})=-2 \mathrm{k} \operatorname{csch}(\mathrm{k} \xi) \\
\mathrm{u}_{1}(\mathrm{x}, \mathrm{t})=-\left[\begin{array}{l}
-\omega \sinh (\mathrm{k} \xi) \cosh ^{2}(\mathrm{k} \xi)+\omega \sinh (\mathrm{k} \xi) \\
+2 \mathrm{k} \cosh ^{3}(\mathrm{k} \xi)-2 \mathrm{k} \cosh (\mathrm{k} \xi)+ \\
2 \mathrm{k}^{2} \mathrm{t} \omega \sinh (\mathrm{k} \xi) \\
/\left[\left(\cosh { }^{2}(\mathrm{k} \xi)-1\right) \sinh (\mathrm{k} \xi)\right]
\end{array}\right] \\
\mathrm{v}(\mathrm{x}, \mathrm{t})=\frac{-2 \mathrm{k}[\cosh (\mathrm{k} \xi)-1+2 \mathrm{k} \cosh (\mathrm{k} \xi) \mathrm{t} \omega \sinh (\mathrm{k} \xi)]}{(\cosh (\mathrm{k} \xi)-1)}
\end{gathered}
$$

In the same manner, the rest of the components of the iteration formula can be obtained:

$$
\begin{aligned}
& u(x, t)=u(x, t)-\left\{\dot{u}+u u^{\prime}+v^{\prime}\right\} d \tau \\
& v(x, t)=v(x, t)-\left\{\dot{v}+(u v)^{\prime}+u^{\prime \prime}\right\} d \tau
\end{aligned}
$$

The exact solution of MB Eq. 19 already has been obtained in ${ }^{[12]}$ as follows:

$$
\begin{array}{r}
u(x, t)=\omega-2 k \operatorname{coth}(k(\xi-\omega t)) \\
v(x, t)=-2 k \operatorname{csch}(k(\xi-\omega t))
\end{array}
$$

Special case two: In subjected problem, if $\alpha=0$ and $\beta=1 / 2$, WBK Eq. 1 can be reduced to the Approximate Long Wave (ALW) equation in shallow water:

$$
\begin{aligned}
& u+u u+v+\frac{1}{2} u=0 \\
& v+(u v)-\frac{1}{2} v=0
\end{aligned}
$$

Considering the application of the VIM to the ALW Eq. 32 with the initial conditions ${ }^{[11,12]}$.

$$
\begin{aligned}
& \mathrm{u}(\mathrm{x}, 0)=\omega-\mathrm{k} \operatorname{coth}(\mathrm{k} \xi) \\
& \mathrm{v}(\mathrm{x}, 0)=-\mathrm{k} \operatorname{csch}(\mathrm{k} \xi)
\end{aligned}
$$

Its correction variational functional in $x$ and $t$ can be expressed respectively as follows:

$$
\begin{aligned}
& u(x, t)=u(x, t)+\lambda(\tau)\left\{\begin{array}{c}
\dot{u}+u u^{\prime}+ \\
v^{\prime}+\frac{1}{2} u^{\prime \prime}
\end{array}\right\} d \tau \\
& v(x, t)=v(x, t)+\lambda(\tau)\left\{\begin{array}{c}
\dot{v}+(u v)^{\prime} \\
-\frac{1}{2} v^{\prime \prime}
\end{array}\right\} d \tau
\end{aligned}
$$

The Lagrange multipliers can therefore, be identified as $\lambda_{1}=\lambda_{2}=-1$ and the variational iteration formula is obtained in the form of:

$$
\begin{aligned}
& u(x, t)=u(x, t)-\left\{\begin{array}{c}
\dot{u}+u u^{\prime}+ \\
v^{\prime}+\frac{1}{2} u^{\prime \prime}
\end{array}\right\} d \tau \\
& v(x, t)=v(x, t)-\left\{\begin{array}{c}
\dot{v}+(u v)^{\prime} \\
-\frac{1}{2} v^{\prime \prime}
\end{array}\right\} d \tau
\end{aligned}
$$

An initial approximation $\mathrm{u}(\mathrm{x}, 0)$ and $\mathrm{v}(\mathrm{x}, 0)$ given by Eq. 33, by the above iteration formula 36 and 37 . We can obtain directly the other components as follows:

$$
\begin{aligned}
& u(x, t)=\omega-k \cosh (k \xi) \\
& \operatorname{vo}(x, t)=-k \operatorname{csch}(k \xi)
\end{aligned}
$$

$$
\mathrm{v}_{1}(\mathrm{x}, \mathrm{t})=\frac{-\mathrm{k}^{2}\left(\cosh ^{2}(\mathrm{k} \xi)-1+2 \mathrm{k} \cosh (\mathrm{k} \xi) \mathrm{t} \omega \sinh (\mathrm{k} \xi)\right)}{\left(\cosh ^{2}(\mathrm{k} \xi)-1\right)^{2}}
$$


In the same manner, the rest of the components of the iteration formula can be obtained.

$$
\begin{aligned}
& \mathrm{u}_{2}(\mathrm{x}, \mathrm{t})=\mathrm{u}_{1}(\mathrm{x}, \mathrm{t})-\int_{0}^{\mathrm{t}}\left\{\mathrm{u}_{1}+\mathrm{u}_{1} \mathrm{u}_{1}^{\prime}+\mathrm{v}_{1}+\frac{1}{2} \mathrm{u}_{1}^{\prime \prime}\right\} d \tau \\
& \mathrm{v}_{2}(\mathrm{x}, \mathrm{t})=\mathrm{v}_{1}(\mathrm{x}, \mathrm{t})-\int_{0}^{\mathrm{t}}\left\{\mathrm{v}_{1}+\left(\mathrm{u}_{1} \mathrm{v}_{1}\right)^{\prime}-\frac{1}{2} \mathrm{v}_{1}^{\prime \prime}\right\} d \tau
\end{aligned}
$$

Exact solution of ALW Eq. 32 is as follows ${ }^{[12]}$ :

$$
\begin{aligned}
& \mathrm{u}(\mathrm{x}, \mathrm{t})=\omega-\mathrm{k} \operatorname{coth}(\mathrm{d}(\xi-\omega \mathrm{t})) \\
& \mathrm{v}(\mathrm{x}, \mathrm{t})=-\mathrm{k}^{2} \operatorname{csch}^{2}(\mathrm{k}(\xi-\omega \mathrm{t}))
\end{aligned}
$$

\section{RESULTS AND DISCUSSION}

The solution of Whitham-Broer-Kaup Wave equation is successfully investigated analytically by Variational Iteration Method (VIM). For clarity, also to illustrate some facts directly from obtained solutions, the propagation of discussed wave phenomena have been shown in Fig. 1-6. Figure 1 and 2, respectively, shows the propagation of $\mathrm{u}(\mathrm{x}, \mathrm{t})$ and $\mathrm{v}(\mathrm{x}, \mathrm{t})$ versus time, $\mathrm{t}$ and displacement, $\mathrm{x}$, for the general form of wave equation (Eq. 1) when $\mathrm{k}=0.1, \omega=0.005, \alpha=1.5$, $\beta=1.5, x_{0}=10$. The behavior of propagation of $\mathrm{u}(\mathrm{x}, \mathrm{t})$ and $\mathrm{v}(\mathrm{x}, \mathrm{t})$ for special case 1 (Eq. 19) have been shown in Fig. 3 and 4 respectively. In the same manner the results of $\mathrm{u}(\mathrm{x}, \mathrm{t})$ and $\mathrm{v}(\mathrm{x}, \mathrm{t})$ for special case 2 (Eq. 32)

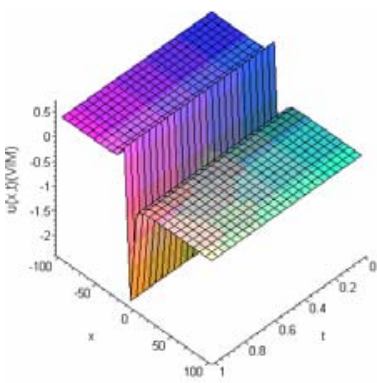

(a)

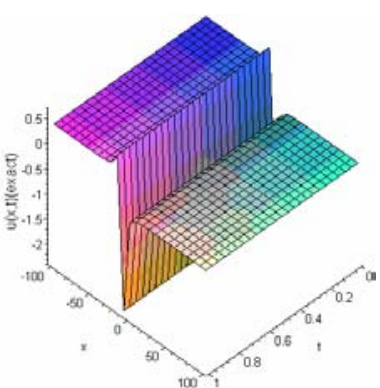

(b)
Fig. 1: Propagation of $u(x, t)$ for Eq. 1, VIM (a) and exact solution (b) for $\mathrm{k}=0.1, \omega=0.005$, $\alpha=1.5, \beta=1.5, x_{0}=10$

are shown in Fig. 5 and 6 respectively. In all figures, (a) refers to VIM and (b) refers to the exact solution. These figures illustrate well agreement between the VIM and exact solution.
For further verification, the error of Fig. 1-6a are shown in Table 1-3 in comparison to those of exact solution. These tables clearly confirm the high accuracy of obtained solutions.

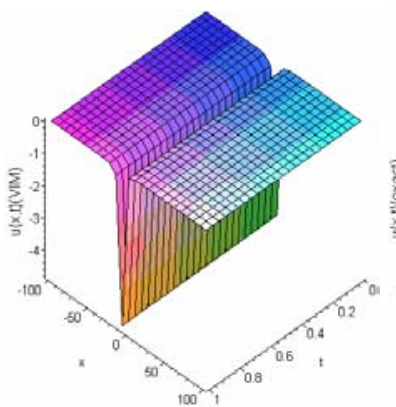

(a)

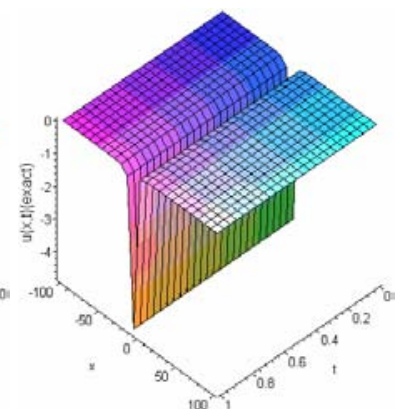

(b)
Fig. 2: Propagation of $v(x, t)$ for Eq. 1, VIM (a) and exact solution (b) for $\mathrm{k}=0.1, \omega=0.005$, $\alpha=1.5, \beta=1.5, x_{0}=10$

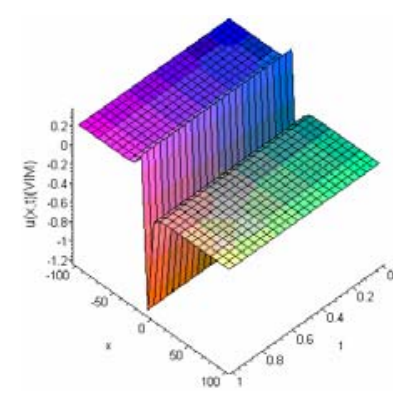

(a)

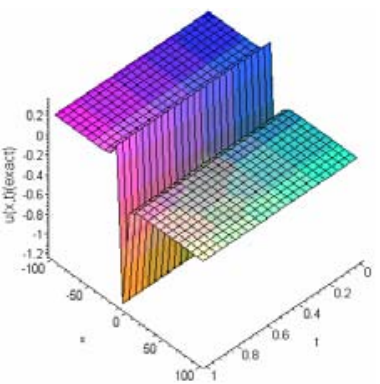

(b)
Fig. 3: Propagation of $\mathrm{u}(\mathrm{x}, \mathrm{t})$ for Eq. 19, VIM (a) and exact solution (b) for $\mathrm{k}=0.1, \omega=0.005$, $\alpha=1.5, \beta=0, x_{0}=10$

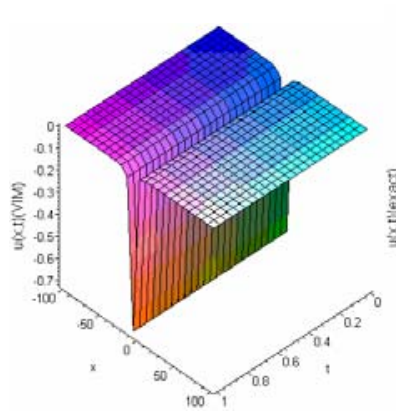

(a)

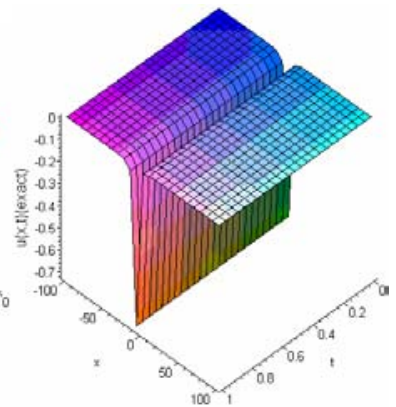

(b)
Fig. 4: Propagation of $v(x, t)$ for Eq. 19, VIM (a) and exact solution (b) for $\mathrm{k}=0.1, \omega=0.005$, $\alpha=1.5, \beta=0, \mathrm{x}_{0}=10$ 
Am. J. Engg. \& Applied Sci., 1 (2): 161-167, 2008

Table 1: The numerical results for $\left|\mathrm{u}_{\text {exact }}-\mathrm{u}_{\mathrm{VIM}}\right|=\psi$ and $\left|\mathrm{v}_{\text {exact }}-\mathrm{v}_{\mathrm{VIM}}\right|=\varphi$ in comparison with the exact solution $(17,18)$ for $\mathrm{u}(\mathrm{x}, \mathrm{t})$ and $\mathrm{v}(\mathrm{x}, \mathrm{t})$ for the approximate solution of the WBK Eq. 1

\begin{tabular}{|c|c|c|c|c|c|c|}
\hline $\mathrm{x} / \mathrm{t}$ & 0 & 0.2 & 0.4 & 0.6 & 0.8 & 1 \\
\hline \multicolumn{7}{|c|}{$\left|\mathrm{u}_{\text {exact }}-\mathrm{u}_{\mathrm{VIM}}\right|=\psi$} \\
\hline-100 & $-5.5 \mathrm{E}-10$ & $-1.3 \mathrm{E}-10$ & -1.5 E-10 & $-5.2 \mathrm{E}-10$ & $-1.0 \mathrm{E}-10$ & -1.0 E-10 \\
\hline-80 & 0.0 & 0.0 & -4.2 E- 10 & 0.0 & 0.0 & -1.0 E-10 \\
\hline-60 & $-2.1 \mathrm{E}-10$ & $-1.4 \mathrm{E}-10$ & -1.7 E-10 & 2.6 E-10 & -4.0 E-10 & -4.0 E-10 \\
\hline-40 & -9.4 E-10 & $-1.0 \mathrm{E}-10$ & $-4.3 \mathrm{E}-10$ & -8.3 E-10 & -5.1 E-10 & -6.2 E-10 \\
\hline-20 & $2.0 \mathrm{E}-10$ & -7.0 E-10 & -6.0 E-10 & 6.9 E-10 & 1.1 E-9 & $3.3 \mathrm{E}-10$ \\
\hline 0 & -5.3 E-10 & -1.5 E-10 & $-1.2 \mathrm{E}-10$ & -7.5 E-10 & -1.2 E-9 & 2.6 E-9 \\
\hline 20 & 5.0 E-10 & 1.3 E-10 & 7.0 E-10 & 5.1 E-10 & 7.2 E-10 & $8.2 \mathrm{E}-10$ \\
\hline 40 & 2.4 E-10 & $2.1 \mathrm{E}-10$ & 2.5 E-10 & 2.6 E-10 & -1.7 E-10 & $-1.2 \mathrm{E}-10$ \\
\hline 60 & $5.1 \mathrm{E}-1$ & 3.4 E-10 & -1.3 E-10 & 2.5 E-10 & 2.3 E-10 & $-2.0 \mathrm{E}-10$ \\
\hline 80 & $5.6 \mathrm{E}-10$ & $5.6 \mathrm{E}-10$ & $5 \mathrm{E}-10$ & $5.1 \mathrm{E}-10$ & $5.9 \mathrm{E}-10$ & $5.1 \mathrm{E}-10$ \\
\hline 100 & 0.0 & 0.0 & 0.0 & 0.0 & 0.0 & 0.0 \\
\hline \multicolumn{7}{|c|}{$\left|\mathrm{v}_{\text {exact }}-\mathrm{v}_{\mathrm{VIM}}\right|=\varphi$} \\
\hline-100 & 3.0 E-18 & $-1.6 \mathrm{E}-12$ & -3.2 E-14 & $-4.8 \mathrm{E}-12$ & -6.4 E-12 & $-8.1 \mathrm{E}-12$ \\
\hline-80 & -3.0 E-16 & -8.8 E-11 & -1.7 E-10 & -2.6 E-10 & -3.5 E-10 & -4.4 E-10 \\
\hline-60 & $1.0 \mathrm{E}-14$ & -4.8 E-9 & -9.6 E-9 & -1.4 E-8 & -1.9 E-8 & -2.4 E-8 \\
\hline-40 & $1.0 \mathrm{E}-12$ & $-2.6 \mathrm{E}-7$ & $-5.3 \mathrm{E}-7$ & -7.9 E-7 & -1.0 E-6 & -1.3 E-6 \\
\hline-20 & $1.0 \mathrm{E}-10$ & -2.5 E-5 & $-5.0 \mathrm{E}-5$ & -7.5 E-5 & $-1.0 \mathrm{E}-4$ & $-1.2 \mathrm{E}-4$ \\
\hline 0 & $1.0 \mathrm{E}-12$ & $2.5 \mathrm{E}-5$ & $5.0 \mathrm{E}-5$ & $7.5 \mathrm{E}-5$ & $1.0 \mathrm{E}-4$ & $1.2 \mathrm{E}-4$ \\
\hline 20 & 1.0 E-14 & $2.6 \mathrm{E}-7$ & 5.3 E-7 & $7.9 \mathrm{E}-7$ & $1.0 \mathrm{E}-6$ & $1.3 \mathrm{E}-6$ \\
\hline 40 & -3.0 E-16 & 4.8 E-9 & 9.6 E-9 & $1.4 \mathrm{E}-8$ & $1.9 \mathrm{E}-8$ & $2.4 \mathrm{E}-8$ \\
\hline 60 & 3.0 E-16 & 8.8 E-11 & 1.7 E-10 & 2.6 E-10 & 3.5 E-10 & $4.4 \mathrm{E}-10$ \\
\hline 80 & $3.0 \mathrm{E}-18$ & $1.6 \mathrm{E}-12$ & 3.2 E-12 & $4.8 \mathrm{E}-12$ & 6.4 E-12 & $8.1 \mathrm{E}-12$ \\
\hline 100 & -1.0 E-19 & $2.9 \mathrm{E}-14$ & $5.9 \mathrm{E}-14$ & 8.9 E-14 & $1.1 \mathrm{E}-13$ & $1.4 \mathrm{E}-13$ \\
\hline
\end{tabular}

Table 2: The numerical results for $\left|\mathrm{u}_{\text {exact }}-\mathrm{u}_{\mathrm{VIM}}\right|=\psi$ and $\left|\mathrm{v}_{\text {exact }}-\mathrm{v}_{\mathrm{VIM}}\right|=\varphi$ in comparison with the exact solution $(30,31)$ for $\mathrm{u}(\mathrm{x}, \mathrm{t})$ and $\mathrm{v}(\mathrm{x}, \mathrm{t})$ for the approximate solution of the MB Eq. 19

\begin{tabular}{|c|c|c|c|c|c|c|}
\hline $\mathrm{x} / \mathrm{t}$ & 0 & 0.2 & 0.4 & 0.6 & 0.8 & 1 \\
\hline \multicolumn{7}{|c|}{$\left|\mathrm{u}_{\text {exact }}-\mathrm{u}_{\mathrm{VIM}}\right|=\psi$} \\
\hline-100 & -1.0 E-10 & $-1.0 \mathrm{E}-10$ & -1.0 E-10 & -1.0 E-10 & -1.0 E-10 & $-1.0 \mathrm{E}-10$ \\
\hline-80 & 0.0 & 0.0 & 3.0 E-10 & 3.0 E-10 & $3.0 \mathrm{E}-10$ & $5.0 \mathrm{E}-10$ \\
\hline-60 & 0.0 & 3.7 E-10 & 7.3 E-9 & $1.0 \mathrm{E}-8$ & $1.4 \mathrm{E}-8$ & $1.8 \mathrm{E}-8$ \\
\hline-40 & -5.2 E-10 & $1.9 \mathrm{E}-7$ & $3.9 \mathrm{E}-7$ & $5.9 \mathrm{E}-7$ & 7.9 E-7 & 9.9 E-7 \\
\hline-20 & -4.4 E-10 & $1.4 \mathrm{E}-5$ & $2.8 \mathrm{E}-5$ & 4.3 E-5 & 5.7 E-5 & 7.2 E-5 \\
\hline 0 & 2.1 E-10 & $1.4 \mathrm{E}-5$ & $2.8 \mathrm{E}-5$ & $4.3 \mathrm{E}-5$ & 5.7 E-5 & $7.2 \mathrm{E}-5$ \\
\hline 20 & 3.8 E-10 & $1.9 \mathrm{E}-7$ & 3.9 E-7 & $5.9 \mathrm{E}-7$ & 7.9 E-7 & 9.9 E-7 \\
\hline 40 & 0.0 & 3.7 E-9 & 7.3 E-9 & $1.0 \mathrm{E}-8$ & $1.4 \mathrm{E}-8$ & $1.8 \mathrm{E}-8$ \\
\hline 60 & $1.0 \mathrm{E}-10$ & $1.0 \mathrm{E}-10$ & 2.3 E-10 & 2.9 E-10 & 3.7 E-10 & $4.5 \mathrm{E}-10$ \\
\hline 80 & $1.0 \mathrm{E}-10$ & $1.0 \mathrm{E}-10$ & $1.0 \mathrm{E}-10$ & $1.0 \mathrm{E}-10$ & 1.0 E-10 & $1.0 \mathrm{E}-10$ \\
\hline 100 & $-1.0 \mathrm{E}-10$ & $-1.0 \mathrm{E}-10$ & $-1.0 \mathrm{E}-10$ & $-1.0 \mathrm{E}-10$ & $-1.0 \mathrm{E}-10$ & $-1.0 \mathrm{E}-10$ \\
\hline \multicolumn{7}{|c|}{$\left|\mathrm{v}_{\text {exact }}-\mathrm{v}_{\mathrm{VIM}}\right|=\varphi$} \\
\hline-100 & $1.0 \mathrm{E}-18$ & $-2.4 \mathrm{E}-13$ & -4.8 E-13 & -7.3 E-13 & -9.7 E-13 & $-1.2 \mathrm{E}-12$ \\
\hline-80 & -3.1 E-17 & $-1.3 \mathrm{E}-11$ & $-2.6 \mathrm{E}-11$ & -3.9 E-11 & $-5.3-11$ & -6.6 E-11 \\
\hline-60 & $1.0 \mathrm{E}-15$ & $-7.20 \mathrm{E}-10$ & -1.4 E-9 & -2.1 E-9 & -2.9 E-9 & -3.6 E-9 \\
\hline-40 & 2.7 E-13 & $-4.0 \mathrm{E}-8$ & $-8.0 \mathrm{E}-8$ & $-1.2 \mathrm{E}-7$ & -1.6 E-7 & $-2.0 \mathrm{E}-7$ \\
\hline-20 & $1.6 \mathrm{E}-11$ & $-3.8 \mathrm{E}-5$ & -7.6 E-5 & $-1.1 \mathrm{E}-5$ & -1.5 E-5 & $-1.8 \mathrm{E}-5$ \\
\hline 0 & $1.3 \mathrm{E}-11$ & $3.8 \mathrm{E}-5$ & 7.6 E-5 & $1.1 \mathrm{E}-5$ & $1.5 \mathrm{E}-5$ & $1.9 \mathrm{E}-5$ \\
\hline 20 & $2.2 \mathrm{E}-13$ & $4.0 \mathrm{E}-8$ & $8.0 \mathrm{E}-8$ & $1.2 \mathrm{E}-7$ & $1.6 \mathrm{E}-7$ & $2.0 \mathrm{E}-7$ \\
\hline 40 & $1.1 \mathrm{E}-15$ & 7.2 E-10 & 1.4 E-9 & 2.1 E-9 & 2.9 E-9 & 3.6 E-9 \\
\hline 60 & -3.3 E-17 & $1.3 \mathrm{E}-11$ & 2.6 E-11 & 3.9 E-11 & $5.3 \mathrm{E}-11$ & $6.6 \mathrm{E}-11$ \\
\hline 80 & 1.6 E-18 & 2.4 E-13 & $4.8 \mathrm{E}-13$ & 7.3 E-13 & 9.7 E-13 & $1.2 \mathrm{E}-12$ \\
\hline 100 & -2.4 E-20 & 4.4 E-15 & 8.9 E-15 & 1.3 E-14 & 1.7 E-14 & 2.2 E-14 \\
\hline
\end{tabular}


Am. J. Engg. \& Applied Sci., 1 (2): 161-167, 2008

Table 3: The numerical results for $\left|\mathrm{u}_{\text {exact }}-\mathrm{u}_{\mathrm{VIM}}\right|=\psi$ and $\left|\mathrm{v}_{\text {exact }}-\mathrm{v}_{\mathrm{VIM}}\right|=\varphi$ in comparison with the exact solution (43, 44) for $u$ and $v$ for the approximate solution of the ALW Eq. 32

\begin{tabular}{|c|c|c|c|c|c|c|}
\hline$x / t$ & 0 & 0.2 & 0.4 & 0.6 & 0.8 & 1 \\
\hline \multicolumn{7}{|c|}{$\left|\mathrm{u}_{\text {exact }}-\mathrm{u}_{\mathrm{VIM}}\right|=\psi$} \\
\hline-100 & 0.0 & 0.0 & 0.0 & 0.0 & 0.0 & 0.0 \\
\hline-80 & 0.0 & 0.0 & -1.0 E-10 & 0.0 & 0.0 & -2.3 E-10 \\
\hline-60 & 0.0 & $-3.1 \mathrm{E}-10$ & -1.1 E9 & 2.5 E-9 & -4.7 E-9 & -7.3 E-9 \\
\hline-40 & -3.2 E-10 & $-1.6 \mathrm{E}-8$ & $-6.6 \mathrm{E}-8$ & $-1.4 \mathrm{E}-7$ & $-2.6 \mathrm{E}-7$ & -4.1 E-7 \\
\hline-20 & 0.0 & -4.8 E-6 & -1.9 E-5 & -4.3 E-5 & -7.7 E-5 & -1.2 E-4 \\
\hline 0 & -1.0 E-10 & -4.8 E-6 & 1.9 E-6 & $4.3 \mathrm{E}-5$ & 7.7 E-5 & $1.2 \mathrm{E}-4$ \\
\hline 20 & 1.9 E-10 & $1.6 \mathrm{E}-8$ & $6.6 \mathrm{E}-8$ & 1.4 E-7 & $2.6 \mathrm{E}-7$ & 4.1 E-7 \\
\hline 40 & 0.0 & 3.4 E-10 & $1.1 \mathrm{E}-9$ & 2.6 E-9 & 4.6 E-9 & 7.2 E-9 \\
\hline 60 & 3.0 E-11 & $7.2 \mathrm{E}-11$ & $1.1 \mathrm{E}-11$ & 7.6 E-11 & $1.5 \mathrm{E}-10$ & $1.3 \mathrm{E}-10$ \\
\hline 80 & 1.0 E-10 & $1.0 \mathrm{E}-10$ & $1.0 \mathrm{E}-10$ & $1.0 \mathrm{E}-10$ & $1.0 \mathrm{E}-10$ & $1.1 \mathrm{E}-10$ \\
\hline 100 & $-5.0 \mathrm{E}-11$ & $-5.0 \mathrm{E}-11$ & $-5.1 \mathrm{E}-11$ & $-5.3 \mathrm{E}-11$ & -5.7 E-11 & -5.9 E-11 \\
\hline \multicolumn{7}{|c|}{$\left|\mathrm{v}_{\text {exact }}-\mathrm{v}_{\mathrm{VIM}}\right|=\varphi$} \\
\hline-100 & 9.7 E-12 & $4.8 \mathrm{E}-12$ & 9.7 E-12 & $1.4 \mathrm{E}-11$ & $1.9 \mathrm{E}-11$ & 2.4 E-11 \\
\hline-80 & 5.3 E-10 & 2.6 E-10 & $5.3 \mathrm{E}-10$ & 7.9 E-10 & 1.0 E-9 & 1.3 E-9 \\
\hline-60 & 2.9 E-8 & $1.4 \mathrm{E}-8$ & $2.9 \mathrm{E}-8$ & 4.3 E-8 & $5.8 \mathrm{E}-8$ & 7.2 E-8 \\
\hline-40 & $1.6 \mathrm{E}-6$ & $8.0 \mathrm{E}-7$ & $1.6 \mathrm{E}-6$ & 2.4 E-6 & $3.2 \mathrm{E}-6$ & 4.0 E- 6 \\
\hline-20 & $2.4 \mathrm{E}-4$ & $1.2 \mathrm{E}-4$ & $2.4 \mathrm{E}-4$ & $3.6 \mathrm{E}-4$ & 4.9 E-4 & $6.1 \mathrm{E}-4$ \\
\hline 0 & $2.4 \mathrm{E}-4$ & $1.2 \mathrm{E}-4$ & $2.4 \mathrm{E}-4$ & $3.6 \mathrm{E}-4$ & $4.9 \mathrm{E}-4$ & $6.1 \mathrm{E}-4$ \\
\hline 20 & $1.6 \mathrm{E}-6$ & $8.0 \mathrm{E}-7$ & $1.6 \mathrm{E}-6$ & 2.4 E-6 & $3.2 \mathrm{E}-6$ & 4.0 E-6 \\
\hline 40 & $2.9 \mathrm{E}-8$ & $1.4 \mathrm{E}-8$ & $2.9 \mathrm{E}-8$ & $4.3 \mathrm{E}-8$ & $5.8 \mathrm{E}-8$ & 7.2 E-8 \\
\hline 60 & 5.3 E-10 & $2.6 \mathrm{E}-10$ & $5.3 \mathrm{E}-10$ & 7.9 E-10 & 1.0 E-9 & 1.3 E-9 \\
\hline 80 & 9.7 E-12 & 4.8 E-12 & 9.7 E-12 & $1.4 \mathrm{E}-11$ & $1.9 \mathrm{E}-11$ & $2.4 \mathrm{E}-11$ \\
\hline 100 & 1.7 E-13 & 8.9 E-14 & $1.7 \mathrm{E}-13$ & $2.6 \mathrm{E}-13$ & $3.5 \mathrm{E}-13$ & 4.4 E-13 \\
\hline
\end{tabular}

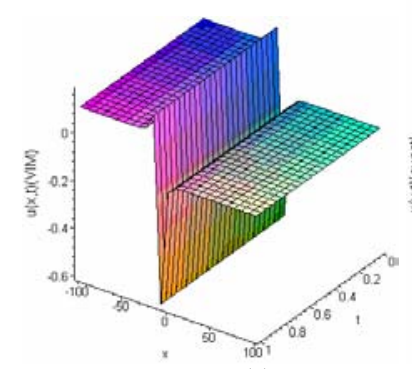

(a)

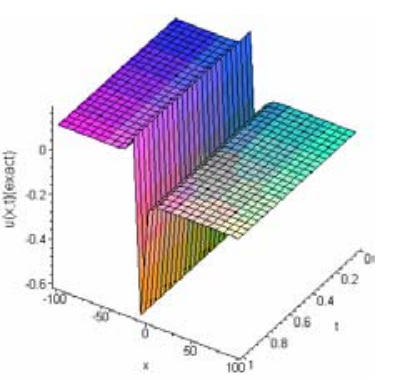

(b)

Fig. 5: Propagation of $u(x, t)$ for Eq. 32, VIM (a) and exact solution (b) for $\mathrm{k}=0.1, \omega=0.005, \alpha=0$, $\beta=1.2, x_{0}=10$

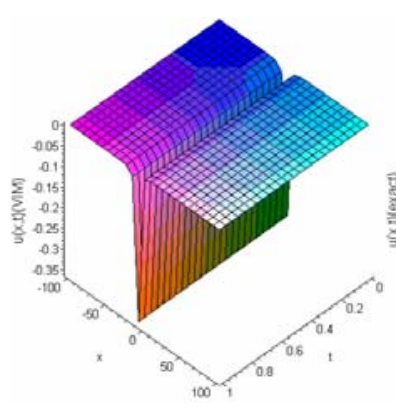

(a)

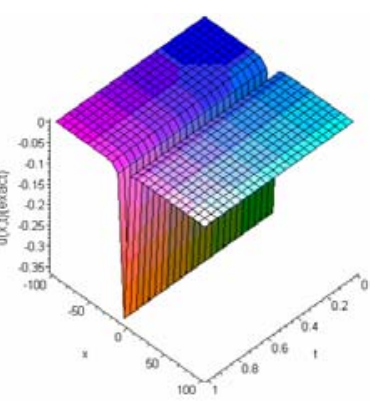

(b)

Fig. 6: Propagation of $v(x, t)$ for Eq. 32, VIM (a) and exact solution (b) for $\mathrm{k}=0.1, \omega=0.005, \alpha=0$, $\beta=1.2, x_{0}=10$

\section{CONCLUSION}

In this research, He's variational iteration method has been successfully implemented to find the analytical solution of nonlinear Whitham-Broer-Kaup (WBK), Modified Boussinesq (MB) and Approximate Long Wave (ALW) equations. All the examples show that the results of the present method are in excellent agreement with those of the exact solutions. Some of the advantages of proposed approach are freely choosing initial conditions with some unknown parameters, so it is capable to achieve the unknown parameters in the initial solution. It also is capable to converge to correct results with fewest number of iterations or even once, for some cases.

\section{REFERENCES}

1. He, J.H., 2006. Some asymptotic methods for strongly nonlinear equations. Int. J. Mod. Phys B., 20: 1141-1199.

URL:http://db0.worldscinet.com/worldscistaging/d etail.nsp

ISSN: 0217-9792 (print version),

ISSN: 1793-6578 (online version), 
2. He, J.H. and X.H. Wu, 2006. Construction of solitary solution and compacton-like solution by variational iteration method. Chaos Soliton Fract., 29: 108-113.

URL:http://www.sciencedirect.com/science?_ob= MImg\&_imagekey=B6TJ44HVDYM181R\&_cdi= $5300 \&$ user $=1400147 \&$ orig $=$ search\& coverDate $=07 \% 2 \mathrm{~F} 31 \% 2 \mathrm{~F} 2006 \& \_\mathrm{sk}=999709998 \& \mathrm{view}=\mathrm{c} \&$ wchp $=$ dGLbVtbzSkWb\&md5=77ba0fc67d1f68ce 1 3f65ca6e 106a601\&ie=/sdarticle.pdf

DOI:10.1016/j.chaos.2005.10.100

ISSN: 0960-0779

3. He, J.H., 2007. Variational iteration method-some recent results and new interpretations. J Computat. Applied Math., 207: 3-17

URL:http://www.sciencedirect.com/science?_ob= MImg\&_imagekey=B6TYH4KSD85631\&_cdi $=56$ $19 \&$ user $=1400147 \&$ orig $=$ search\&_coverDate $=1$ 0\%2F01\%2F2007\&_sk $=997929998 \&$ view $=$ c\&wch

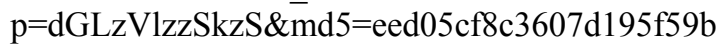
52ce3f8af7e\&ie=/sdarticle.pdf

DOI:10.1016/j.cam.2006.07.009

ISSN: 0377-0427

4. Tolou, N., D.D. Ganji, M.J. Hosseini and Z.Z. Ganji, 2007. Application of homotopy perturbation method in nonlinear heat diffusionconvection-reaction equations. Open Mech. J., 1: $20-25$.

URL:http://www.benthamopen.org/pages/gen.php? file $=20$ TOMECHJ.pdf\&PHPSESSID $=\mathrm{e} 85 \mathrm{f} 974 \mathrm{~b} 83$ f528a29b3e0ab05a13416a

DOI: $10.2174 / 1874158400701010020$

ISSN: $1874-1584$

5. Ganji, D.D. and A. Sadighi, 2007. Application of homotopy-perturbation and variational iteration methods to nonlinear heat transfer and porous media equations. J. Computat. Applied Math., 207: 24-34.

URL:http://www.sciencedirect.com/science?_ob= MImg\&_imagekey=B6TYH4KV8TD411\&_cdi=5 $619 \&$ user $=1400147 \&$ orig $=$ search \& coverDate $=$ 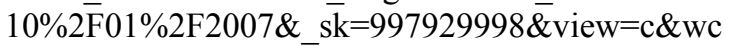

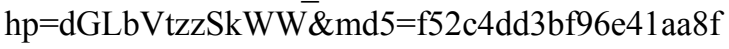
f08358a7e091c\&ie $=/$ sdarticle.pdf

DOI:10.1016/j.cam.2006.07.030

ISSN: 0377-0427

6. Tolou, N., I. Khatami, B. Jafari and D.D. Ganji, 2008. Analytical solution of nonlinear vibrating systems. Am. J. Applied Sci., 5: 1219-1224.

URL:http://www.scipub.org/fulltext/ajas/ajas59121 9-1224.pdf

ISSN: $1812-5654$.
7. Rafei, M.and D.D. Ganji, 2006. Explicit solutions of Helmholtz equation and fifth-order $\mathrm{KdV}$ equation using homotopy perturbation method. Int. J. Nonl. Sci. Numer. Simulat., 7: 321-328. ISSN Number: 1565-1339

8. Ganji, D.D. and A. Sadighi, 2006. Application of He's homotopy-perturbation method to nonlinear coupled systems of reaction-diffusion equations. Int. J. Nonl. Sci. Numer. Simulat., 7: 411-418.

ISSN Number: 1565-1339

9. Khatami, I., N. Tolou, J. Mahmoudi and M. Rezvani, 2008. Application of homotopy analysis method and variational iteration method for shock wave equation. J. Applied Sci., 8: 848-853.

URL:http://www.ansijournals.com/jas/0/848853.pdf ISSN: 1812-5654.

10. Mahmoudi, J., N. Tolou, I. Khatami and D.D. Ganji, 2008. Explicit solution of nonlinear zkbbm wave equation using exp-function method. J. Applied Sci., 8: 358-263.

URL:http://ads.ari.uniheidelberg.de/abs/2008JApS c...8..358M

ISSN: $1812-5654$.

11. Fuding, Xie, Zhenya Yana and Hongqing Zang, 2001. Explicite and exact traveling wave solution of whitham-boer-kaup shallow water equations. Phys. Lett. A., 285: 76-80.

URL:http://www.sciencedirect.com/science?_ob= MImg\&_imagekey=B6TVM43B29C2F35\&_cdi=5 $538 \&$ user $=1400147 \&$ orig $=$ search\&_coverDate $=$ $06 \% 2 \overline{\mathrm{F}} 25 \% 2 \mathrm{~F} 2001 \& \mathrm{k}=997149998 \& \mathrm{view}=\mathrm{c} \& \mathrm{wch}$

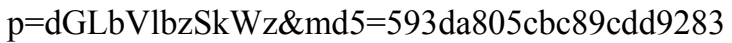
$01539 \mathrm{c} 28 \mathrm{~d} 06 \mathrm{c} \& \mathrm{ie}=/$ sdarticle.pdf

S0375-9601(01)00333-4

ISSN: 0375-9601

12. Salah M. and El-Sayed ,Dogan Kaya, 2005. Exact and numerical traveling wave solutions of whitham-broer-kaup equations. Applied Math. and Computat., 167: 1339-134.

URL:http://www.sciencedirect.com/science?_ob= MImg\&_imagekey=B6TY84DR02TKG15\&_cdi= 5612 \&_user $=1400147 \&$ orig $=$ search\&_coverDate $=08 \% 2 \mathrm{~F} 15 \% 2 \mathrm{~F} 2005 \mathrm{sk}=998329997 \&$ view $=\mathrm{c} \& \mathrm{wch}$

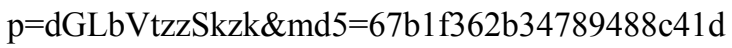
a1 ec40a040e\&ie $=/$ sdarticle.pdf

DOI:10.1016/j.amc.2004.08.012

ISSN: 0096-3003 